\title{
Investigation on the BER performance of downlink JT-CoMP-NOMA with different modulation schemes
}

\author{
Norshidah Katiran ${ }^{1}$, Shaharil Mohd Shah², Noorsaliza Abdullah ${ }^{3}$, Aimi Syamimi Abdul Ghaffar ${ }^{4}$, \\ Faiz Asraf Saparudin 5 \\ ${ }^{1,2,3}$ Faculty of Electrical and Electronic Engineering, Universiti Tun Hussein Onn Malaysia, Malaysia \\ ${ }^{4,5}$ Faculty of Engineering Technology, Universiti Tun Hussein Onn Malaysia, Malaysia
}

\begin{tabular}{l} 
Article Info \\
\hline Article history: \\
Received Feb 3, 2020 \\
Revised Apr 25, 2020 \\
Accepted May 12, 2020 \\
\hline Keywords: \\
BER \\
JT-CoMP-NOMA \\
Modulation schemes \\
Spectral efficiency \\
Time-frequency resources \\
\end{tabular}

\begin{abstract}
The principle of joint transmission coordinated multipoint with nonorthogonal multiple access (JT-CoMP-NOMA) is to allow multiple cells in the network to cooperatively transmit data to users using the same network resources in frequency and time domains. The approach would be beneficial in enhancing the system performance in the context of spectral efficiency. This paper presents the performance comparison of JT-CoMP-NOMA for several modulation schemes including QPSK, 16-QAM and 64 QAM in downlink transmission in the context of bit error rate. We conduct an investigation to compare the error performance of JT-CoMP-NOMA with NOMA system. Our simulation results demonstrate that the JT-CoMP-NOMA provides lower bit error rate compared to NOMA system.
\end{abstract}

Copyright (c) 2020 Institute of Advanced Engineering and Science. All rights reserved.

\section{Corresponding Author:}

Norshidah Katiran,

Faculty of Electrical and Electronic Engineering,

Universiti Tun Hussein Onn Malaysia,

86400 Parit Raja, Batu Pahat, Johor, Malaysia.

Email: norshida@uthm.edu.my

\section{INTRODUCTION}

The fifth generation $(5 \mathrm{G})$ mobile communication networks are envisioned to provide much higher data rates and enhanced user throughput under higher connectivity density. Coordinated Multipoint (CoMP) is one of the primary technologies for LTE-Advanced that improves system spectral efficiency and realized through base station coordination. In CoMP systems, the available system resources are allocated among active users in a coordinated way. Several types of CoMP transmission schemes have been suggested including joint transmission (JT) and coordinated beamforming coordinated scheduling (CB/CS). For JT-CoMP, useful information such as data, channel state information (CSI) and scheduling decision are made available across multiple cells in the network. Multiple base stations (BSs) in the network cooperatively transmit data to users located at the cell boundary to reduce inter-cell interference and thus enhance the total system throughput [1-6]. On the other hand, in the CoMP with CS/CB, data for a user is transmitted from one BS for a timefrequency resource. However, scheduling/beamforming decision is coordinated among cells in CoMP. Under this scenario, transmit beamforming weights for each individual user are generated to diminish the unwanted interference to other users scheduled within the CoMP cells [7-8]. As a result, the received signal to interference noise ratio (SINR) and the cell edge user throughput can be improved. In a system that applies the orthogonal multiple access (OMA) [9], a channel dedicated to a cell edge user cannot be used for other users' transmission at the same time [10]. Hence, the system spectral efficiency degrades with the increasing number of cell edge users [11]. In recent times, there have been investigations on advanced multiple access schemes such as nonorthogonal multiple access (NOMA) which overcome the limitations addressed in OMA schemes [12-17]. 
The concept of NOMA as illustrated in Figure 1 is to carry out simultaneous transmission to multiple users over the same resources. In power-domain NOMA, a serving cell schedules more than one user to transmit using the same resources by superposing their signals in the power domain [18]. The superposition is realized such that each NOMA user is able to decode the desired signal. Therefore, NOMA is anticipated as a promising approach to enhance the spectral efficiency of $5 \mathrm{G}$ mobile communication systems.

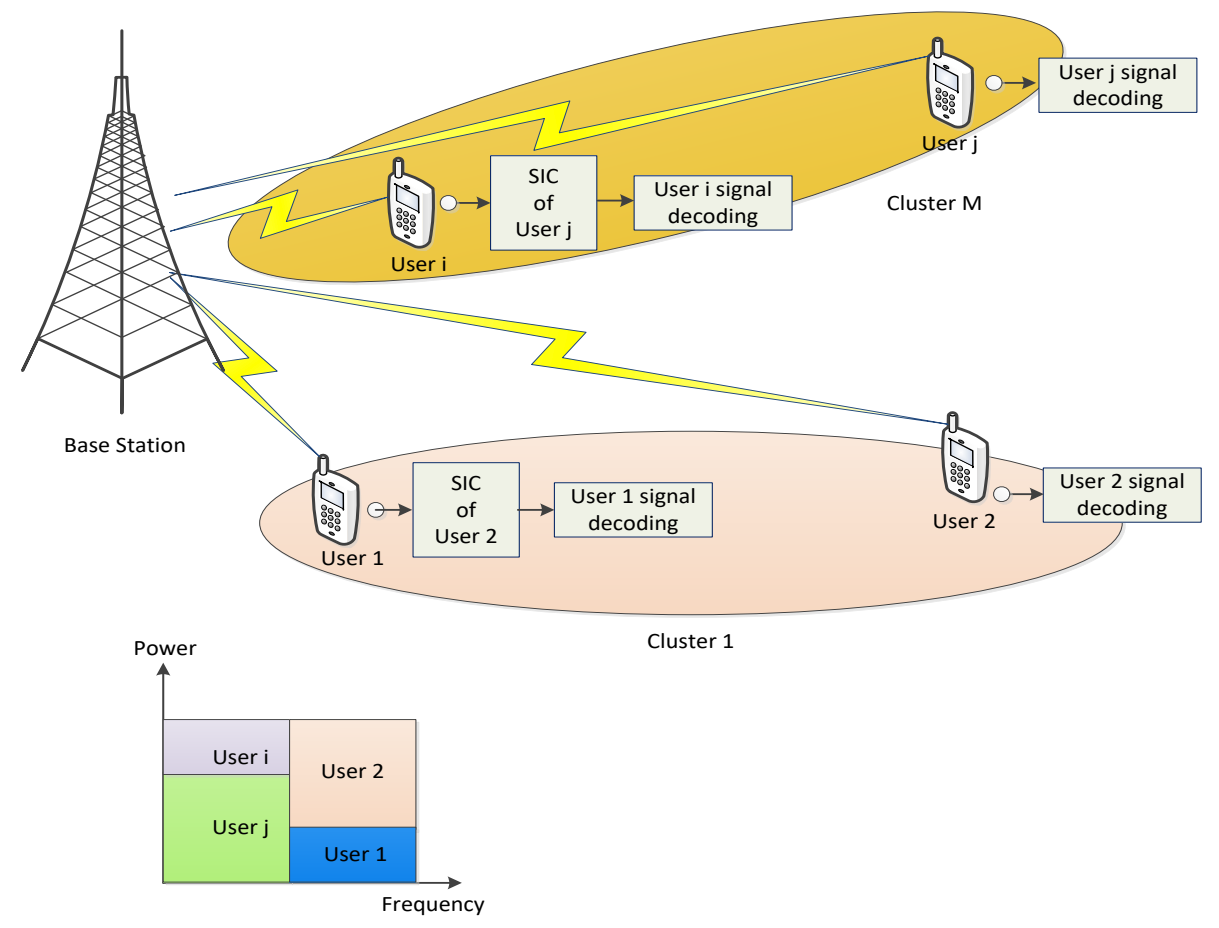

Figure 1. Principle of NOMA through exploitation of signal power diversity

Attaining good error performance is one of vital goals of a network operator as it guarantees smooth application delivery to end user devices. On the other hand, achieving high transmission rate is always desirable from network operator perspective. One conventional method to achieve high data rates is the use of higherorder modulation, which is capable to increase the data rate within a fixed bandwidth [19-23]. In LTE and LTE-Advanced systems, QPSK, 16-QAM and 64-QAM are being adopted for the symbol modulation of orthogonal frequency division multiplexing (OFDM) [24]. Previous study in [25] has shown that 64-QAM provides the lowest bit error rate (BER) and best suited for downlink NOMA compared to QPSK and 16-QAM. Motivated by these observation, in this paper we study the error performance of JT-CoMP-NOMA and compare with NOMA system.

Several modulation schemes are adopted, including QPSK, 16-QAM and 64-QAM. The simulation results show that the BER of JT-CoMP-NOMA system is lower compared to NOMA system [25] under different modulation schemes. The rest of the paper is outlined as follows. Section II describes the JT-CoMPNOMA system model for downlink transmission and Section III provides the BER performance of the system with different modulation formats. Finally, the concluding remarks are drawn in Section IV.

\section{RESEARCH METHOD}

\subsection{User Clustering Algorithm}

Figure 2 shows a JT-CoMP-NOMA system model considered in this paper. The system consists of $M$ BSs having $N_{T}$ transmit antennas and $K$ mobile users with $N_{R}$ receive antennas deployed in each cell. The users in each cell are divided into $L$ clusters and each cluster contains two users. Within a cluster, the user close to the BS with larger channel gain is defined as strong user, $u_{\text {near }}$, while the user far from the BS with smaller channel gain is known as weak user, $u_{\text {far }}$. 


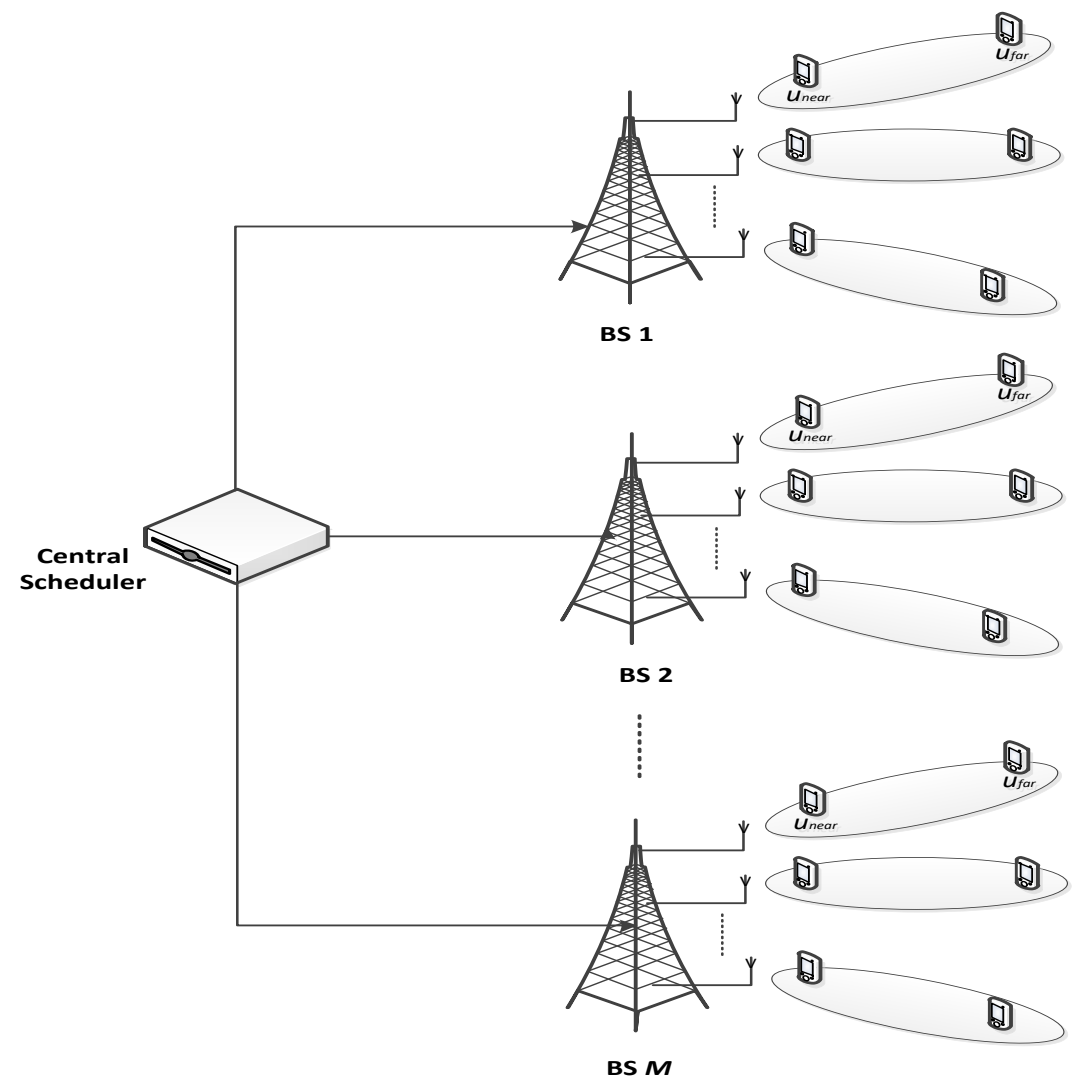

Figure 2. Downlink JT-CoMP-NOMA System

\subsection{Power Allocation Algorithm}

In NOMA, the serial interference cancellation (SIC) procedure is performed at the receiver of $u_{\text {near }}$. This means that, $u_{f a r}$ will treat the interference caused by $x_{\text {near }}$ as unknown interference and next will directly decode its own data, $x_{f a r}$. On the other hand, $u_{\text {near }}$ will first decode the data vector $x_{f a r}$ by considering the interference caused by $x_{\text {near }}$ as unknown interference and then subtract $x_{f a r}$ from received signal and decode $x_{\text {near }}$ from the remain part of received signal.

The message signals targeting to $u_{\text {near }}$ and $u_{f a r}$ are superimposed over physical resource block (PRB) $r$, with transmit power $\alpha P_{l}$ and $(1-\alpha) P_{l}$ for $u_{\text {near }}$ and $u_{f a r}$, respectively. From literature, it has been shown that the SIC performance deteriorates when the power difference between the $u_{\text {near }}$ and $u_{f a r}$ within the same cluster becomes smaller [26-28]. According to [28], if the power allocation factors of both users has a difference of less than 0.35 , the SIC process at $u_{\text {near }}$ becomes imperfect and the data recovery process at $u_{\text {far }}$ is badly affected. In view of that, in our study, we fixed the power factor of $u_{\text {near }}$ and $u_{f a r}$ at values 0.35 and 0.65 , respectively.

The BSs in the JT-CoMP-NOMA system are connected to a central scheduler which has the responsibility to manage available transmission resources in a coordinated way. In order to reduce the stringent requirements on backhaul link, selective combining (SC) is employed in this paper, where only one BS is selected by the central scheduler to serve the user. The SC receiver decides one BS that provides the best signalto-noise ratio (SNR) value.

\section{RESULTS AND DISCUSSION}

In the simulation study, we investigate the performance of downlink JT-CoMP-NOMA system with different modulation schemes including QPSK, 16-QAM and 64-QAM and make comparison with NOMA system [24]. The simulation settings adopted in the study are tabulated in Table 1.

Figure 3 shows the BER performance of $u_{\text {near }}$ in the system when QPSK, 16-QAM and 64-QAM modulation schemes are applied. Simulation results in Figure 3 clearly shows that the JT-CoMP-NOMA system gains the lowest BER when 64-QAM is adopted compared to QPSK and 16-QAM modulation schemes. 
The BER trend is similar with the results obtained in [25]. On the other hand, the error performance of $u_{f a r}$ adopting QPSK, 16-QAM and 64-QAM is shown in Figure 4, which demonstrates the same trends as for $u_{\text {near }}$. We observed that that BER performance can be improved significantly when 64-QAM modulation scheme is adopted at both users as proved by [25].

Table 1. Simulation parameters

\begin{tabular}{cc}
\hline Parameters & Assumptions \\
\hline Cell Layout & Hexagonal 3 cell CoMP system \\
Channel Model & Rayleigh fading model \\
Radius of BS coverage & $500 \mathrm{~m}$ \\
BS transmit antenna & 4 \\
User terminat receive antenna & 2 \\
BS transmit power & $43 \mathrm{dBm}$ \\
Scheduling interval & $1 \mathrm{~ms}$ \\
PRB bandwidth & $180 \mathrm{kHz}$ \\
Path Loss Model & $128.1+37.6 \log (d$ in $\mathrm{km}) \mathrm{dB}$ \\
\hline
\end{tabular}

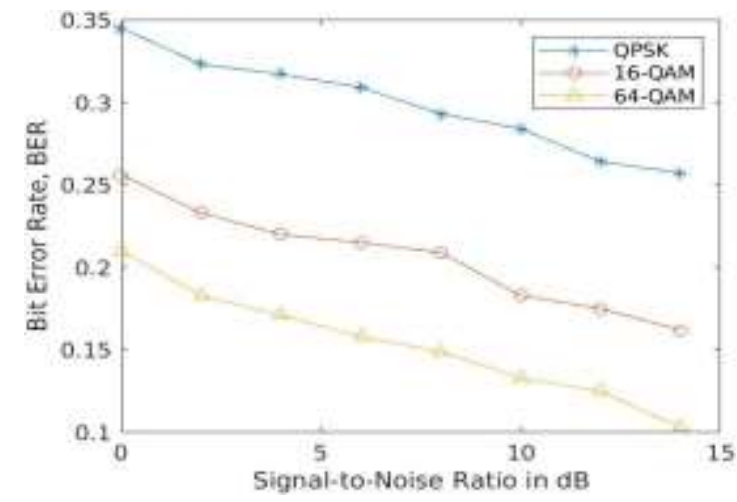

Figure 3. Error performance of $u_{\text {near }}$ with different modulation schemes in JT-CoMP-NOMA

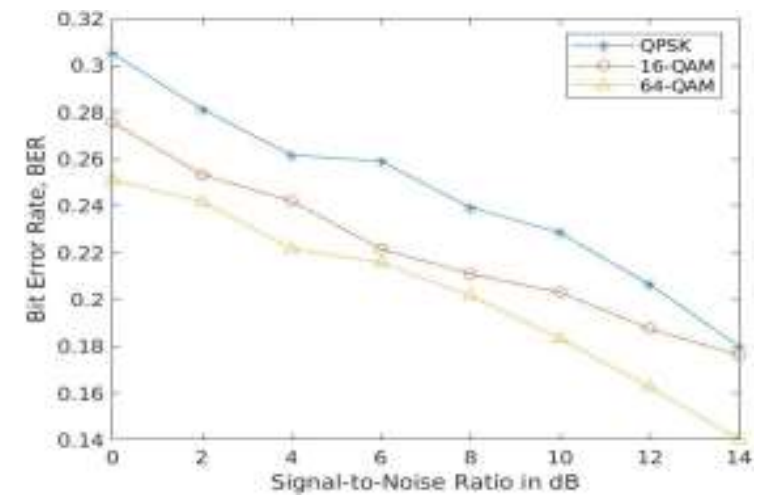

Figure 4. Error performance of $u_{\text {far }}$ with different modulation schemes in JT-CoMP-NOMA

Next, we conducted a comparison study to investigate the performance of JT-CoMP-NOMA and NOMA systems [25], when 64-QAM modulation scheme is applied to both $u_{\text {near }}$ and $u_{\text {near }}$ signals. Numerical results from the study are presented in Figure 5 and Figure 6, respectively. The results in Figure 5 and Figure 6 clearly show that the error performance of both $u_{\text {near }}$ and $u_{\text {far }}$ in JT-CoMP-NOMA are lower as compared to NOMA system. This reflect the additional performance gain obtained by exploiting spatial diversities available in JT-CoMP-NOMA system.

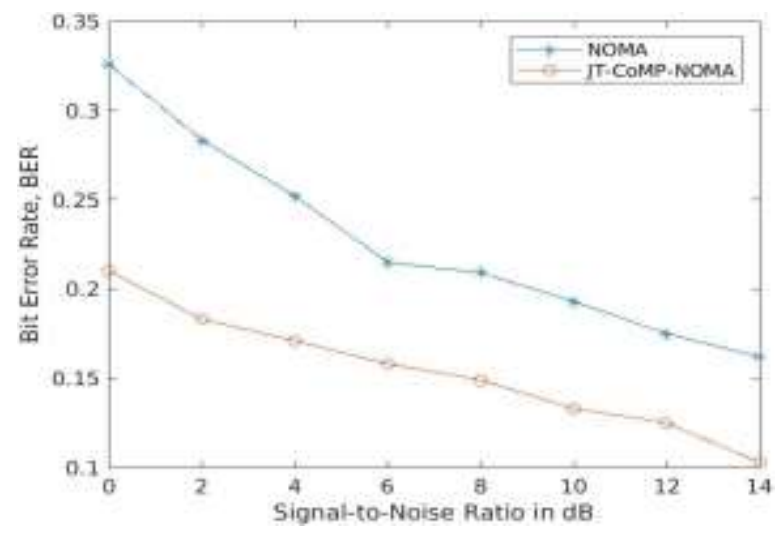

Figure 5. Error performance comparison of $u_{\text {near }}$ in JT-CoMP-NOMA and NOMA systems

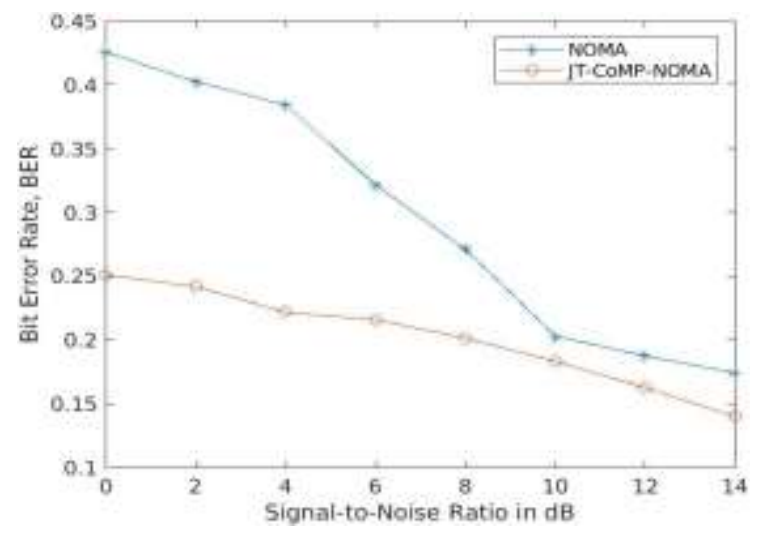

Figure 6. Error performance comparison of $u_{f a r}$ in JT-CoMP-NOMA and NOMA systems 


\section{CONCLUSION}

In this paper, we study the BER performance of JT-CoMP-NOMA system when various modulation scheme are adopted, including QPSK, 16-QAM and 64-QAM. The simulation results indicate that 64-QAM can provide better BER performance in JT-CoMP-NOMA system than QPSK and 16-QAM when employed to both users' signals. In addition, the study shows that BER of JT-CoMP-NOMA system is lower compared to NOMA system due to exploitation of diversities available in the wireless systems.

\section{ACKNOWLEDGEMENTS}

We would like to thank the Ministry of Education, Malaysia and Research Management Centre of Universiti Tun Hussein Onn Malaysia for the financial support under grant code K147.

\section{REFERENCES}

[1] M. Sawahaschi, Y. Kishiyama, A. Morimoto, D. Nishikawa, and M. Tanno, "Coordinated Multipoint Transmission/Reception Techniques for LTE-Advanced," IEEE Wirel. Commun., vol. 17, no. 3, pp. 26-34, 2010.

[2] D. Lee et al., "Coordinated Multipoint Transmission and Reception in LTE-Advanced : Deployment Scenarios and Operational Challenges," Commun. Mag. IEEE, vol. 50, no. 2, pp. 148-155, 2012.

[3] C. Yang, S. Han, X. Hou, and A. F. Molish, "How do we design CoMP to achieve Its Promised Potential?," IEEE Wirel. Commun., vol. 20, no. 1, pp. 67-74, 2013.

[4] H. Touati, H. Castel-Taleb, B. Jouaber, and S. Akbarzadeh, "Model-Based optimization for JT CoMP in C-RAN," INFOCOM 2019 - IEEE Conf. Comput. Commun. Work. INFOCOM WKSHPS 2019, pp. 403-409, 2019.

[5] J. Chen, X. Ge, Y. Zhong, and Y. Li, "A Novel JT-CoMP Scheme in 5G Fractal Small Cell Networks," IEEE Wirel. Commun. Netw. Conf. WCNC, vol. 2019-April, pp. 1-7, 2019.

[6] I. S. Hburi and H. F. Khazaal, "An efficient two-stage user association scheme for green c-RAN systems," Indones. J. Electr. Eng. Comput. Sci., vol. 16, no. 2, pp. 793-802, 2019.

[7] Y. Yang, B. Bai, W. Chen, and L. Hanzo, "A Low-Complexity Cross-Layer Algorithm for Coordinated Downlink Scheduling and Robust Beamforming Under a Limited Feedback Constraint," IEEE Trans. Veh. Technol., vol. 63, no. 1, pp. 107-118, 2014.

[8] W. Ning, T. Zhang, C. Feng, W. Zhao, and Y.-N. Li, "An opportunistic feedback scheme for downlink coordinated scheduling/beamforming," 2012 Comput. Commun. Appl. Conf., pp. 76-80, Jan. 2012.

[9] P. S. Swapna, S. S. Pillai, and K. G. Sreeni, "Resource allocation algorithm for symmetrical services in OFDMA systems,” Indones. J. Electr. Eng. Comput. Sci., vol. 18, no. 2, pp. 867-874, 2020.

[10] Z. M. Abid, A. A. Jafaar, and S. Q. Hadi, "PAPR reduction in OFDM system for DVB-S2," Indones. J. Electr. Eng. Comput. Sci., vol. 17, no. 1, pp. 317-323, 2019.

[11] S. Kanchi, S. Sandilya, D. Bhosale, A. Pitkar, and M. Gondhalekar, "Overview of LTE-A technology," in 2013 IEEE Global High Tech Congress on Electronics, 2013, pp. 195-200.

[12] A. Beylerian, "Coordinated Non-Orthogonal Mulitple Access," 2016.

[13] W. Shin, M. Vaezi, B. Lee, D. J. Love, J. Lee, and H. V. Poor, "Non-orthogonal multiple access in multi-cell networks: Theory, performance, and practical challenges," IEEE Commun. Mag., vol. 55, no. 10, pp. 176-183, 2017.

[14] M. S. Ali, E. Hossain, and D. I. Kim, "Coordinated Multipoint Transmission in Downlink Multi-Cell NOMA Systems: Models and Spectral Efficiency Performance,” IEEE Wirel. Commun., vol. 25, no. 2, pp. 24-31, 2018.

[15] M. S. Ali, E. Hossain, and D. I. Kim, "Non-Orthogonal Multiple Access (NOMA) for Downlink Multiuser MIMO Systems: User Clustering, Beamforming, and Power Allocation," 2016.

[16] M. R. Usman, A. Khan, M. A. Usman, Y. S. Jang, and S. Y. Shin, "On the Performance of Perfect and Imperfect SIC in Downlink Non Orthogonal Multiple Access (NOMA)," no. October, pp. 6-8, 2016.

[17] J. Choi, "Non-orthogonal multiple access in downlink coordinated two-point systems," IEEE Commun. Lett., vol. 18, no. 2, pp. 313-316, 2014.

[18] L. Dai, B. Wang, Y. Yuan, S. Han, C. L. I, and Z. Wang, "Non-orthogonal multiple access for 5G: Solutions, challenges, opportunities, and future research trends," IEEE Commun. Mag., vol. 53, no. 9, pp. 74-81, 2015.

[19] Y. Wang, W. Liu, M. Jin, S. Jang, and J. M. Kim, "FQAM/FPSK modulation for spatial modulation systems," 2016 Int. Conf. Inf. Commun. Technol. Converg. ICTC 2016, pp. 511-515, 2016.

[20] A. F. Aziz, O. A. M. Aly, and U. S. Mohammed, "2019, 36 th National Radio Science Conference (NRSC 2019), Apri 1 16-18, 2019, Port Said, Egypt Arab Academy for Science, Technology and Maritime Transport High Efficiency Modulation Technique For Visible Light Communication (VLC) 2019, 3," 2019 36th Natl. Radio Sci. Conf., no. Nrsc, pp. 100-107, 2019.

[21] A. Mussot et al., "Observation of broken symmetry in the modulation instability recurrence," Opt. InfoBase Conf. Pap., vol. Part F82-C, no. 2001, p. 59000, 2017.

[22] I. Yildirim, E. Basar, and I. Altunbas, "Quadrature channel modulation," IEEE Wirel. Commun. Lett., vol. 6, no. 6, pp. 790-793, 2017.

[23] X. Yao, Y. Ding, and R. Cai, "Modulation Performance Analysis of Terahertz Satellite Communications," 2016 Int. Conf. Netw. Inf. Syst. Comput., no. c, pp. 50-54, 2017.

[24] K. Senda and H. Otsuka, "Downlink Multiuser Superposition Using QPSK and 256-QAM in Mobile Communication Systems," Int. Conf. Ubiquitous Futur. Networks, ICUFN, vol. 2018-July, pp. 385-388, 2018. 
[25] G. Niharika, "Performance comparison of modulation schemes for Downlink NOMA," Proc. 2nd Int. Conf. Inven. Syst. Control. ICISC 2018, no. Icisc, pp. 1431-1433, 2018.

[26] C. Yan, A. Harada, A. Benjebbour, Y. Lan, A. Li, and H. Jiang, "Receiver design for downlink non-orthogonal multiple access (NOMA)," IEEE Veh. Technol. Conf., vol. 2015, 2015.

[27] K. Saito, A. Benjebbour, A. Harada, Y. Kishiyama, and T. Nakamura, "Link-level performance of downlink NOMA with SIC receiver considering error vector magnitude," IEEE Veh. Technol. Conf., vol. 2015, pp. 6-10, 2015.

[28] K. Saito, A. Benjebbour, Y. Kishiyama, Y. Okumura, and T. Nakamura, "Performance and design of SIC receiver for downlink NOMA with open-loop SU-MIMO," 2015 IEEE Int. Conf. Commun. Work. ICCW 2015, pp. 1161$1165,2015$.

\section{BIOGRAPHIES OF AUTHORS}
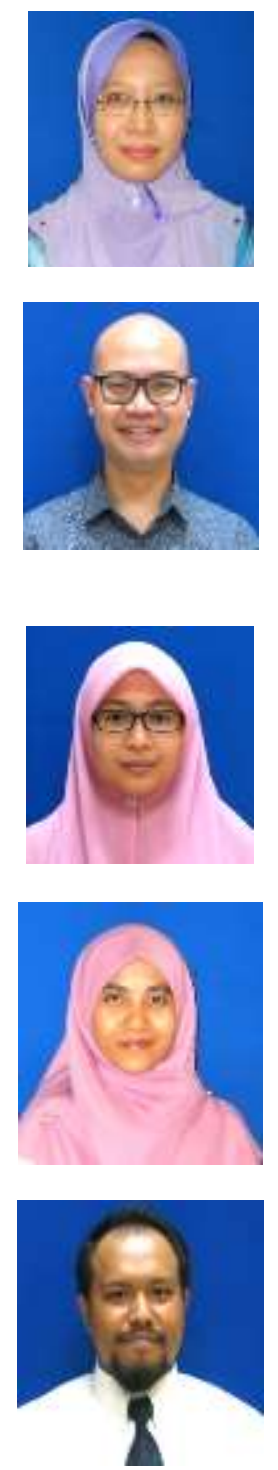

Norshidah Katiran received her Ph.D in Electrical Engineering from Universiti Teknologi Malaysia, in 2015, her M.Eng. degree in Communication and Computer Engineering from Universiti Kebangsaan Malaysia, in 2004, and her B.Eng. degree in Electrical Engineering (Telecommunications) from Universiti Teknologi Malaysia, in 2001. Currently, she is a Senior Lecturer in the Department of Communication Engineering at Universiti Tun Hussein Onn Malaysia. Her major research interests include optimization of resource allocation in cooperative networks and multiple input multiple output (MIMO) transmissions.

Shaharil Mohd Shah obtained his B. Eng in Microwave and Communication from Multimedia University (MMU) in 2002. He received his M.Sc in Microwave Engineering and Wireless Subsystems Design from the University of Surrey, UK in 2004 and pursuing his Ph.D in Communication Engineering from the University of Birmingham, UK before graduating in 2016. $\mathrm{He}$ is currently a senior lecturer in the Department of Communication Engineering, Faculty of Electrical and Electronic Engineering, Universiti Tun Hussein Onn Malaysia (UTHM). His area of research includes, but not limited to, design of microwave devices, active antennas measurement and nonlinear characterisation of active devices.

Noorsaliza Abdullah received B.Eng. and M.Eng degrees in Electronics and Telecommunications from the Universiti Teknologi Malaysia (UTM), Malaysia, in 2003 and 2005, respectively, and her Ph.D. degree from Shizuoka University, Shizuoka, Japan, in 2012. In 2003, she joined Universiti Tun Hussein Onn Malaysia (UTHM), Malaysia, as a Tutor and awarded a scholarship to further her M.Eng. and Ph.D. degrees. Her research interests include array antenna, adaptive beamforming, and mobile communications.

Aimi Syamimi Ab Ghafar received her B.Sc. in Electrical Engineering (Telecommunication) (2010) and $\mathrm{PhD}$ (2015) from Universiti Teknologi Malaysia. Currently, she serves as a lecturer at Universiti Tun Hussein Onn Malaysia. Her current research interests are Next-Generation Mobile Technology, LTE-Advanced, cooperative relay communications, and resource allocation in wireless communication networks.

Faiz Asraf Saparudin received his Bachelor B.Sc. in Electrical Engineering (Telecommunication) with First Class Honours and Doctorate Degree from Universiti Teknologi Malaysia, in 2010 and 2015, respectively. In 2010, he received WAMY Academic Excellence Award. He is currently working as a lecturer in Universiti Tun Hussein Onn Malaysia. His current research interests include radio resource management, distributed algorithms, nature-inspired techniques, multiagent system and game theoretic approach for next-generation mobile network. 